\title{
MIGRAÇÃO VERTICAL E TAXAS FISIOLÓGICAS DE Thalia democratica (SALPIDAE: THALIACEA) NA RESERVA MARINHA DO ARVOREDO, SANTA CATARINA
}

\author{
RESGALLA JR., C.; CARVALHO, J.L.; PEREIRA FO., J.; RÖRIG, L.R.; RODRIGUES- \\ RIBEIRO, M.; TAMANAHA, M.S. \& L.A.O. PROENÇA
}

\author{
CTTMar/UNIVALI - Cx.P. 360, Rua Uruguai 458, Itajaí, SC, 88.302-202, Brasil - \\ cresgalla@univali.br
}

\begin{abstract}
RESUMO
Entre os dias 17 e 18 de dezembro de 1998 foi realizado um fundeio na margem leste da ilha do Arvoredo, dentro da Reserva Biológica Marinha do Arvoredo (27ำ19,6'-278,6' S e 48²7,1'$48^{\circ} 9,1^{\prime}$ W), com o Navio de Pesquisa Soloncy Moura (CEPSUL/IBAMA). Durante o fundeio foram realizados perfis de salinidade e temperatura utilizando um CTD bem como coletadas amostras de água para a determinação do amônio. A biomassa em peso úmido do zooplâcton, fracionada em classes de tamanho, foi coletada por arrastos verticais em 3 estratos na coluna de água. Foram executadas incubações a $5 \mathrm{~m}$ de profundidade, em frasco tipo DBO, de Oozooides da Salpa Thalia democrática com fitoplâncton coletado no mesmo local para as estimativas de suas taxas de filtração e de suas taxas de crescimento pela liberação de blastozooides. Os dados de temperatura e salinidade medidos durante 22 horas não mostraram uma variação temporal significativa, em que a Água Tropical ocorreu em superfície e a Água Subtropical abaixo dos $20 \mathrm{~m}$ de profundidade. A biomassa do zooplâncton apresentou um nítido comportamento de migração vertical, com uma maior concentração no estrato intermediário (10 a 20 m) durante o dia e com um deslocamento para o estrato superficial (0 a $10 \mathrm{~m}$ ) no entardecer. Comportamento similar ocorreu com a concentração de amônio na coluna de água indicando provável processo de regeneração e bioconvecção deste nutriente. A Salpa Thalia democratica foi o organismo dominante na fração maior que $1000 \mu \mathrm{m}$ e a responsável pela maior biomassa nas amostras. Os testes de incubação desta espécie apresentaram uma taxa de filtração média de $176,4 \mathrm{~mL}_{\text {.dia}}{ }^{-1}$ e taxa de crescimento de blastozooides de 8 a 10,3 \% em comprimento por hora.
\end{abstract}

Palavras Chave: Thalia democrática, Ilha do Arvoredo, Santa Catarina, Fisiologia, Migração vertical.

\section{VERTICAL MIGRATION AND PHYSIOLOGICAL RATES OF THE Thalia democratica (SALPIDAE: THALIACEA) IN ARVOREDO BIOLOGICAL MARINE RESERVE, SANTA CATARINA}

\begin{abstract}
Between December 17th and 18th of 1998the Soloncy Moura Research Vessel (CEPSUL/ IBAMA) was stationed for 24 hours at the eastern margin of the Arvoredo island, inside of the Arvoredo Biological Marine Reserve $\left(27^{\circ} 19,6^{\prime}-27^{\circ} 8,6^{\prime} \mathrm{S}\right.$ and $\left.48^{\circ} 27,1^{\prime}-48^{\circ} 9,1^{\prime} \mathrm{W}\right)$. During this time the temperature and salinity profiles were obtained using a CTD, and water samples were collected for ammonium determination. The biomass in wet weight of the size classed zooplankton, was collected by vertical trawls in 3 strata in the water column. Incubations were executed at 5 meters depth in DBO flasks, of the Salpidae Thalia democratica Oozooids and the phytoplankton collected in the same place. The filtration rates were estimated by the increase of feopigments and growth rates by blastozooids liberation. The temperature and salinity data measured along the 24 hours
\end{abstract}


didn't show a significant temporal variation, with the Tropical Water occurring at surface and the Subtropical Water below 20 meters depth. The zooplankton biomass presented a clear vertical migration behaviour, with larger concentrations in the intermediary stratum (10 to $20 \mathrm{~m}$ ) during the day and with a displacement for the superficial stratum $(0$ to $10 \mathrm{~m})$ at sunset. The ammonium concentration exhibited a similar behaviour in the water column indicating probable regeneration processes and bioconvection of this nutrient. The species Thalia democratica was the dominant organism in the fraction larger than $1000 \mu \mathrm{m}$ and it was responsible for the highest zooplankton biomass in the samples. The incubation experiments of this species presented an average filtration rate of $176.4 \mathrm{~mL}$.day ${ }^{-1}$ and growth rate of blastozooids from 8 to $10.3 \%$ in length per hour.

Keywords: Thalia democratica, Arvoredo Island, Santa Catarina State, physiology, vertical migration.

\section{INTRODUÇÃO}

As Salpas são organismos holoplanctônicos abundantes na costa sul e sudeste brasileira, ocorrendo principalmente no fim do verão e início do outono. Segundo PiresVanin et al. (1993) e Resgalla Jr. et al. (2001) estes organismos ocorrem em manchas e estão associados a regiões de ressurgências no embaiamento de São Paulo e sul do Brasil. Estas manchas, constituídas praticamente pela espécie Thalia democratica apresenta alta taxas de crescimento individual e populacional (Heron, 1972a e 1972b), o que ocasiona altos valores de biomassa zooplanctônica para a região (Meneghetti, 1973).

Somado a isto, estes organismos são também caracterizados por altas taxas de filtração e baixas de assimilação, fazendo com que exerçam forte influência sobre a ecologia pelágica, pois privam o desenvolvimento de outros herbívoros por competição e alteram a estrutura de tamanho dos produtores primários pelo empacotamento do fitoplâncton acelerando sua deposição para o fundo por meio de pelotas fecais (Andersen \& Nival, 1988; Blackburn, 1979 e Esnal, 1981). As salpas podem atuar como bombas biológicas na remoção do $\mathrm{CO}_{2}$ fixado pelo fitoplâncton como mecanismo compensatório das alterações climáticas mundiais. Segundo Gibbs (1995), o aumento das populações destes organismos nas últimas décadas é um indicativo indireto do aquecimento do planeta devido a maior disponibilidade do $\mathrm{CO}_{2}$ na atmosfera.
A costa de Santa Catarina tem sido pouco investigada quanto a seus constituintes planctônicos (Brandini et al., 1997 e Valentin et al., 1994), mas a sua geomorfologia recortada, presença de ilhas e ventos dominantes de nordeste tem proporcionado condições de ocorrência de processos de ressurgências de curta duração (Carvalho et al., 1998). Tentar relacionar a ocorrência de salpas e medidas de suas taxas fisiológicas em resposta a processos de ressurgências foi o objetivo deste trabalho que envolveu um fundeio de 24 horas próximo a ilha do Arvoredo (SC). Além disto, o conhecimento gerado sobe a ecologia deste importante constituinte do sistema pelágico será fundamental para o entendimento dos processos biológicos a que estão sujeitos a costa sul e sudeste do Brasil.

\section{MATERIAL E MÉTODOS}

Entre os dias 17 e 18 de dezembro de 1998 foi realizado um fundeio na margem leste da ilha do Arvoredo, dentro da Reserva Biológica Marinha do Arvoredo $\left(27^{\circ} 19,6^{\prime}-27^{\circ} 8,6^{\prime} \mathrm{S}\right.$ e $48^{\circ} 27,1^{\prime}-48^{\circ} 9,1^{\prime}$ W), com o Navio de Pesquisa Soloncy Moura (CEPSUL/IBAMA) (Fig. 1). Durante o fundeio de 24 horas, a cada hora, foram realizados perfis verticais de salinidade e temperatura utilizando um CTD Sensordata. Amostras de água foram obtidas com o auxílio de garrafas de amostragem tipo Niskin, em superfície, 10, 20 e $35 \mathrm{~m}$ de profundidade a cada 4 horas. 
As amostras de água foram filtradas em filtro de fibra de vidro Whatman GF/F imediatamente após a coleta, divididas em alíquotas e congeladas para posterior determinação do amônio $\left(\mathrm{NH}_{4}^{+}\right)$e o material retido nos filtros destinado para a determinação de pigmentos. O amônio foi determinado pela formação do complexo colorido azul de indofenol, corrigido pelo efeito da salinidade, conforme método descrito em Strickland \& Parsons (1972). O complexo colorido foi lido em espectrofotômetro duplo-feixe (Shimadzu UV 160 A) em cubeta de $5 \mathrm{~cm}$ de trajeto óptico.

A concentração da clorofila a (cla a) e produtos de degradação foi determinada por meio de cromatografia líquida de alta eficiência (CLAE), segundo o método descrito em Wright et al. (1991). A extração foi feita em 2 a $3 \mathrm{~mL}$ de acetona $90 \%$, em refrigerador por 24 horas. A cromatografia ocorreu numa coluna FLD-ODS $4 \mu \mathrm{m}$ ( $4.5 \mathrm{~cm}$ x $4.5 \mathrm{~mm}$, Shimadzu) e os pigmentos detectados por fotodiodo de varredura (SPD M10A) e fluorímetro (RF551) alinhados em série. Os dados foram adquiridos por um computador carregado com o programa da LC10 da Shimadzu. A cla a foi calibrada em função da resposta do padrão (SIGMA). Os produtos de degradação foram separados em dois tipos básicos: feoforbídeos e feofitinas, conforme sua eluição anterior ou posterior a cla a, respectivamente (Barlow et al. 1993). A concentração dos produtos de degradação foi obtida a partir da resposta de fluorescência da cla a acidificada, considerando um fator de correção para perda de massa, $\mathrm{PM}=592$ para feoforbídeos e PM 870 para feofitinas.

Amostras de zooplâncton foram obtidas mediante arrastos verticais utilizando uma rede WP-2 de $200 \mu \mathrm{m}$ de tamanho de malha, equipada com fluxômetro e dispositivo de fechamento. Os arrastos foram executados a cada 4 horas e em três estratos da coluna de água (0-10, 10-20 e 20-35 m) sendo o material coletado fixado em formol a $4 \%$. A biomassa em peso úmido do zooplâncton, fracionada em três classes de tamanho (> 2000, 2000-1000 e

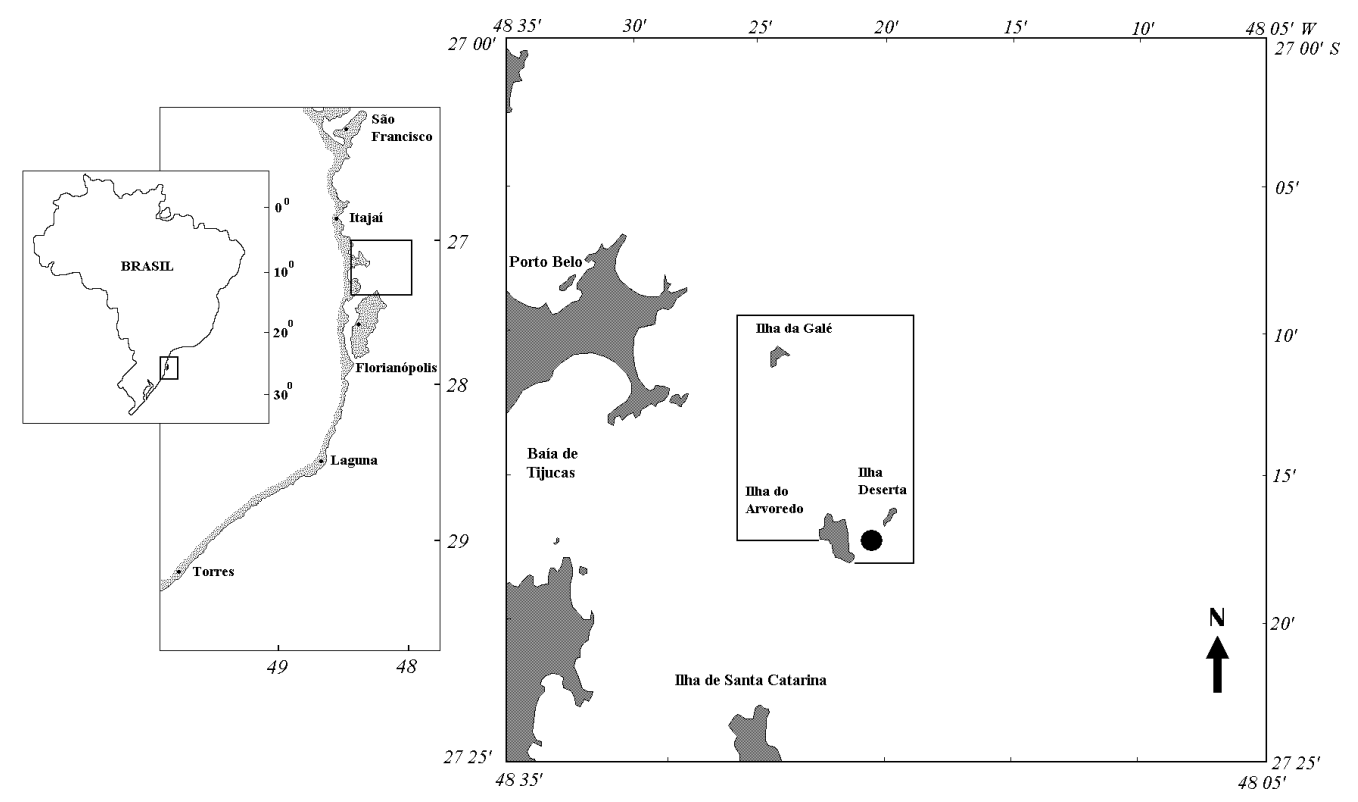

Figura 1 - Área de estudo com a localização do ponto do fundeio $(\bullet)$. 
1000-200 $\mu \mathrm{m}$ ) foram estimadas segundo as recomendações de Beers (1976).

Para as estimativas da taxa de filtração e crescimento da salpa Thalia democratica foram realizadas incubações de 12 horas, em frascos tipo DBO de $300 \mathrm{~mL}$, de Oozooides com estolão e blastozooides em desenvolvimento. Nos frascos teste foi inoculado fitoplâncton coletado no mesmo local com rede de $25 \mu \mathrm{m}$ previamente filtrado em malha de $200 \mu \mathrm{m}$. Os experimentos foram executados em cinco réplicas e mais dois controles sem exemplares de salpas. Os organismos foram coletados em arrastos adicionais abrangendo toda a coluna de água e com a sua transferência individual para os frascos teste (DBO) com o auxílio de conta-gotas de boca larga. Os organismos foram aleatoriamente sorteados, sendo 5 fixados e destinados para análise biométrica para o tempo inicial e 5 destinados para as incubações. Os frascos de DBO foram fixados em estrutura plástica que os mantinha na horizontal e fixados em cabo a $5 \mathrm{~m}$ de profundidade do local do fundeio da embarcação.

As taxas de filtração foram estimadas pelo incremento de feopigmentos medidos por cromatografia líquida de alta eficiência (CLAE) segundo método apresentado para amostras de água. Para isto a água dos frascos teste foi filtrada em filtro de fibra de vidro Whatman GF/ $\mathrm{F}$ a bordo e congelados. As estimativas das taxas de filtração foram realizadas pelo método modificado de Omori \& Ikeda (1984):

$$
T F=\frac{V}{N}\left\{\left[\frac{\left(\operatorname{Ln} C_{t 1}-\operatorname{Ln} C_{t 0}\right)}{\Delta T}\right]-f\right\}
$$

sendo:

$\mathrm{TF}=$ Taxa de filtração $(\mathrm{mL}$. dia-1)

$\mathrm{C}_{\mathrm{t} 1}=$ Concentração de feopigmentos final

$\mathrm{C}_{\mathrm{t} 0}=$ Concentração de feopigmentos inicial

$\mathrm{V}=$ Volume do frasco teste $(\mathrm{mL})$

$\mathrm{N}=$ Número de organismos por frasco teste

$\Delta \mathrm{T}=$ Intervalo de tempo do teste (dia) $f=$ fator de correção de feopigmentos calculado pela mesma fórmula para os fracos controle.

As taxas de crescimento de Thalia democratica foram estimadas pela variação no número e tamanho de blatozooides liberados durante o período de incubação a partir da separação e fixação em formol a 4 \% dos organismos presentes nos frascos no fim dos experimentos. Medidas de comprimento foram realizadas em microscópio estereoscópico com ocular micrométrica. Os pesos dos organismos foram estimados mediante a relação comprimento $X$ peso seco da espécie (Fig. 2).

\section{RESULTADOS E DISCUSSÃO}

Os perfis de temperatura e salinidade realizados durante o fundeio não mostraram uma variação temporal significativa (Fig. 3), cuja Água Tropical (AT) ocorreu até os $20 \mathrm{~m}$ de profundidade e a Água Subtropical (AST) predominou abaixo desta profundidade. Devido aos ventos de quadrante oeste observado durante o período amostral, não foi observada uma elevação da Água Subtropical que pudesse caracterizar um processo de ressurgência.

As variações da cla a e de seus produtos de degradação não apresentaram um pa-

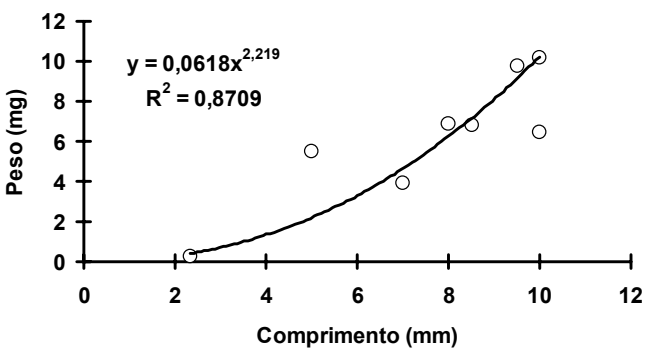

Figura 2 - Relação comprimento $(\mathrm{mm})$ e peso seco de oozoides e blastozooides da salpa Thalia democratica obtido em exemplares fixados. 
drão claro durante o período de estudo. A cla a variou de 89,7 a 683,3 ng/L, com média de 298 ng/L, o que pode ser considerado como valores de biomassa fitoplanctônica baixos para a costa de Santa Catarina (Proença, 2002).

Para o amônio, seu padrão de variação demonstrou um comportamento diferenciado na coluna de água (Fig. 4) com uma mudança dos valores mais elevados (> 20 (Mol/L) de 20 m às 17 horas para $10 \mathrm{~m}$ de profundidade às 21 horas. A presença de amônio em águas marinhas bem oxigenadas está relacionada à atividade biológica. Esta variação coincidiu com a variação da biomassa zooplanctônica, como será vista a seguir, indicando a importância da atividade biológica na regeneração de nutrientes na coluna d'água.
Altos valores de biomassa do zooplâncton foram estabelecidos nos primeiros horários de amostragem do fundeio, sofrendo dispersão com o aumento dos ventos de quadrante oeste ocorridos durante o período. Entretanto, a biomassa do zooplâncton apresentou um nítido comportamento de migração vertical, com uma maior concentração no estrato intermediário (10 a $20 \mathrm{~m}$ ) durante o dia e com um deslocamento para o estrato superficial ( 0 a $10 \mathrm{~m})$ no entardecer (Fig. 5). Para estes horários de coleta, a Salpa Thalia democratica foi o organismo dominante na fração > $2000 \mu \mathrm{m}$ para indivíduos adultos e na fração de 1000 a $2000 \mu \mathrm{m}$ para indivíduos juvenis, sendo a responsável pela maior biomassa nas amostras.
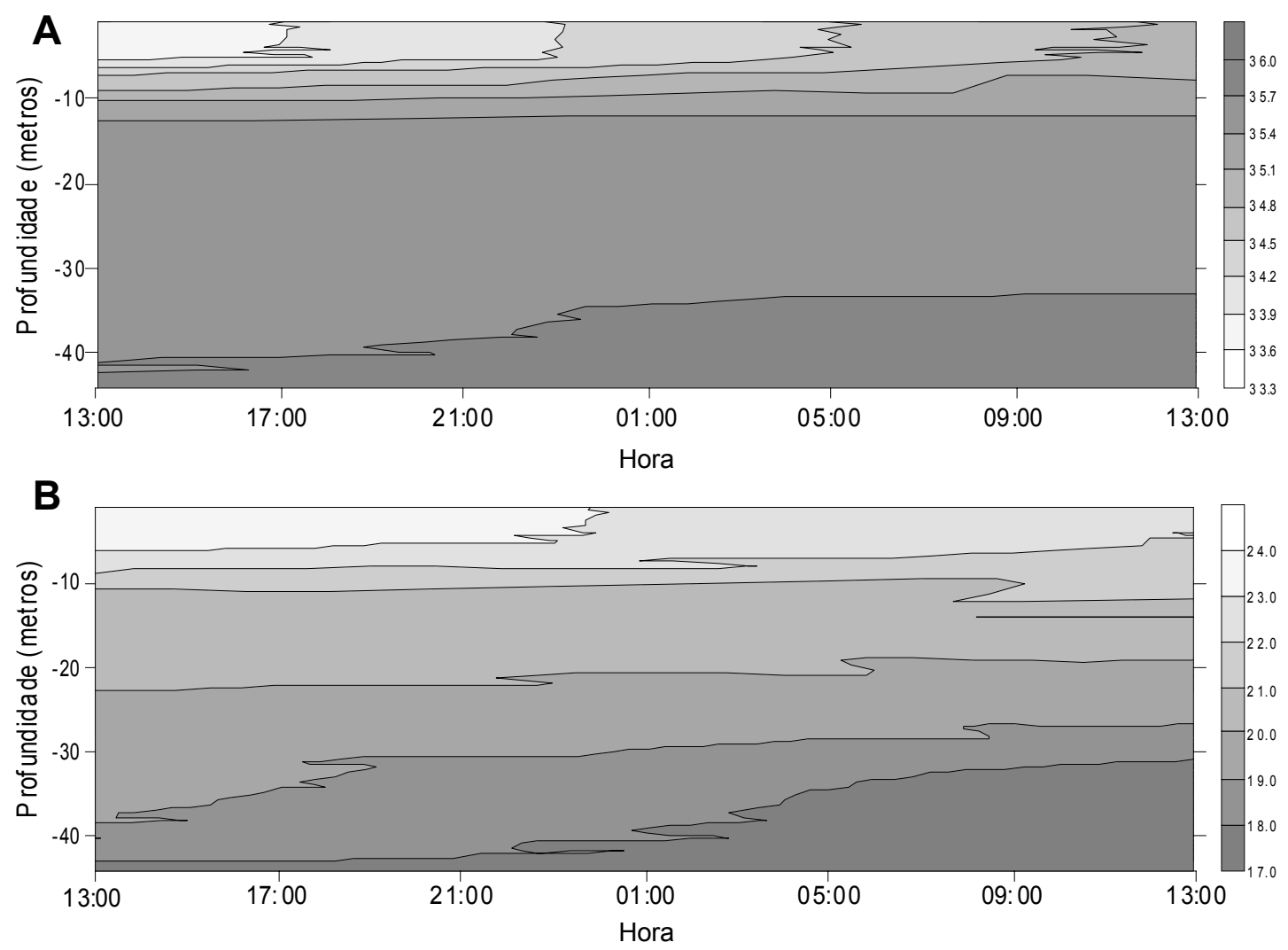

Figura 3 - Variação da temperatura (A) e salinidade (B) na coluna de água coletadas a cada hora por um período de 24 horas. 


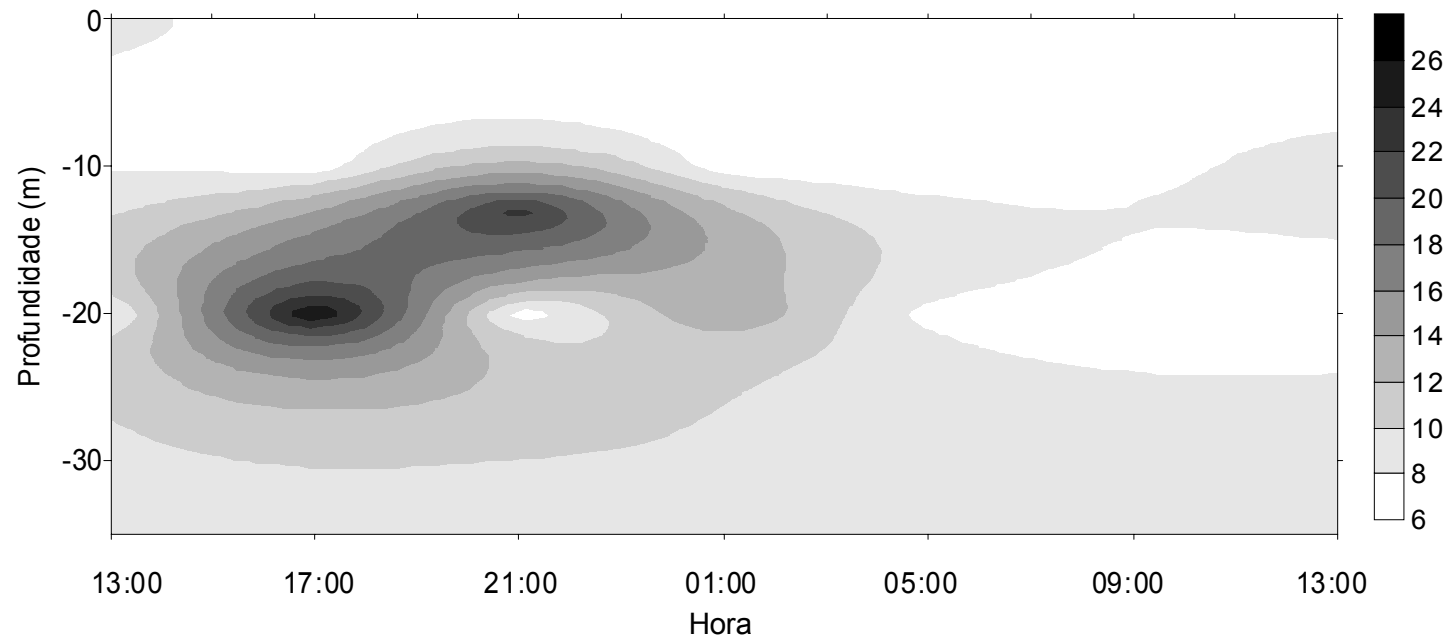

Figura 4 - Variação do $\mathrm{N}-\mathrm{NH}_{4}((\mathrm{~mol} / \mathrm{L})$ na coluna de água coletados a cada 4 horas por 24 horas.
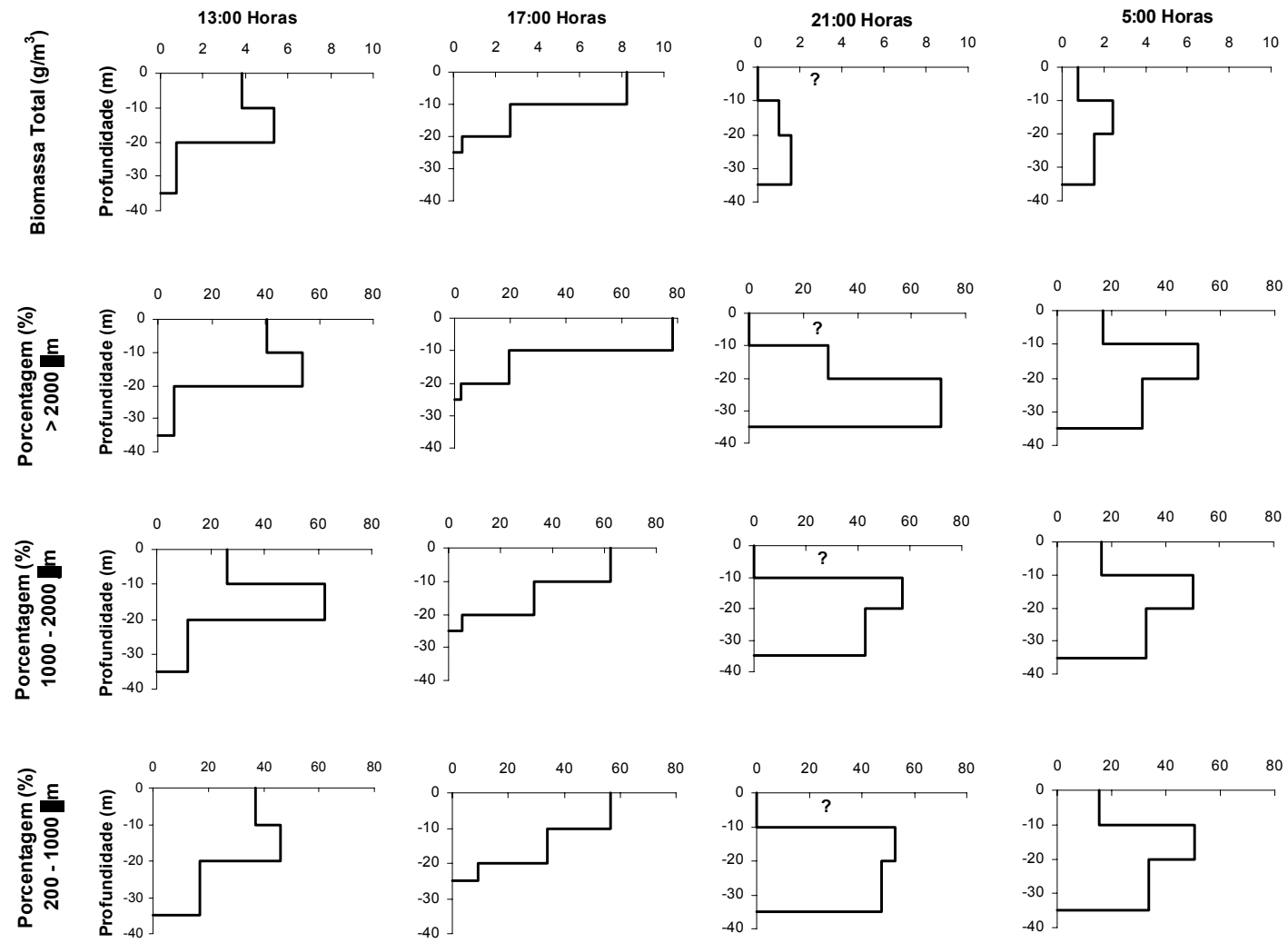

Figura 5 - Biomassa total do zooplâncton em peso úmido e os percentuais por classe de tamanho em três estratos da coluna de água coletados a cada 4 horas ao longo de 15 horas de amostragem. 


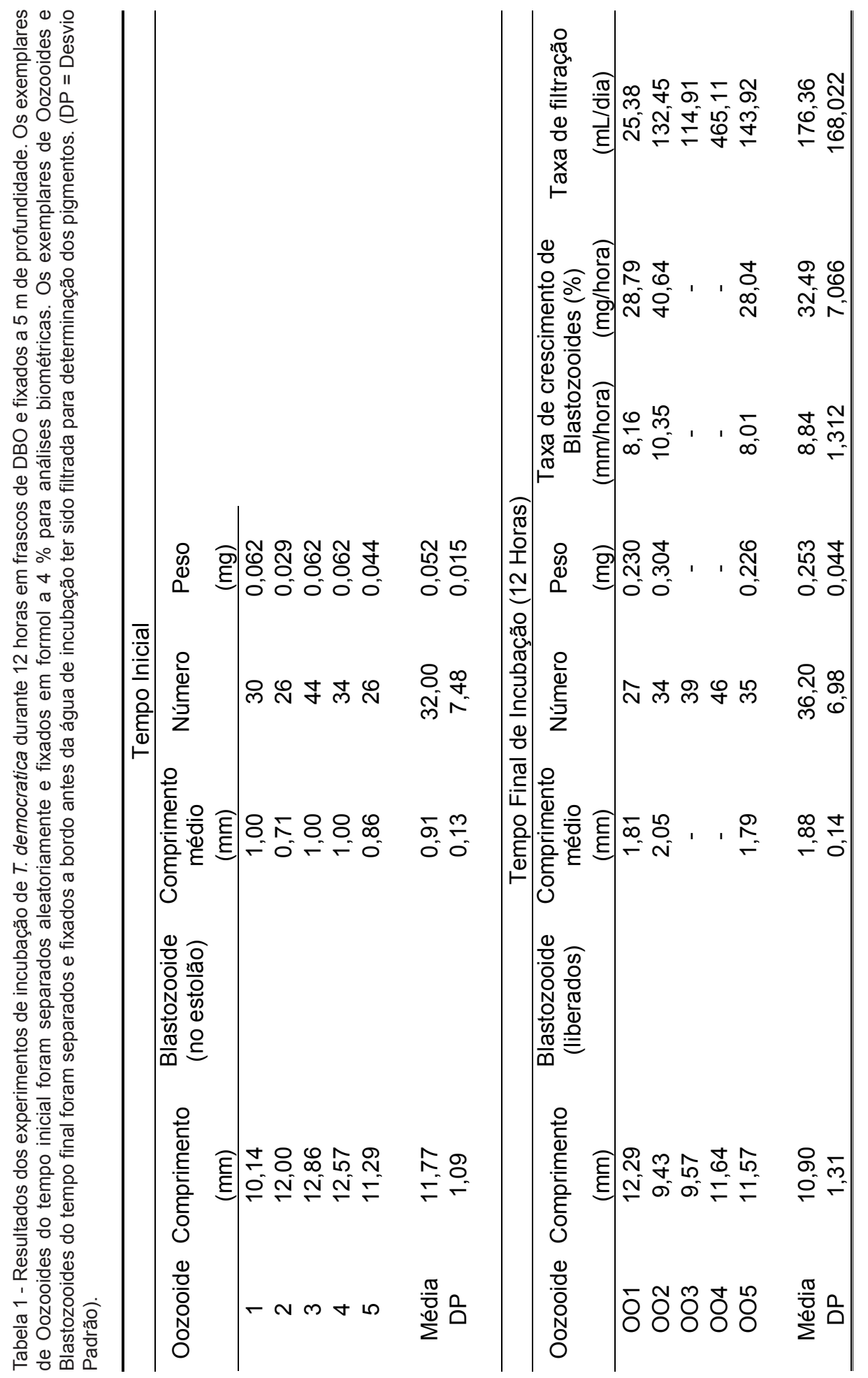


Thalia democratica já foi estudada na Reserva Biológica Marinha do Arvoredo por Fernandes (1998) em novembro de 1995. Foi a única espécie de salpa registrada, apresentando altas densidades (> 7000 org./1000 m3) e com predomínio de formas juvenis indicando reprodução contínua na área. Amaral (1996) e Amaral et al. (1997) destacaram a importância de T. democratica no sul e sudeste do Brasil, com distribuição mais costeira, mas com comportamento de migração inversa ao observado neste trabalho (Amaral et al., 1997).

Os resultados obtidos neste trabalho sugerem que Thalia democratica apresenta atividade alimentar tanto em camadas superficiais como em estratos mais profundos ou subsuperficiais, que, associado aos resultados do amônio, podem favorecer a produção regenerada pelas taxas de excreção, indicando provável processo de regeneração e bioconvecção deste nutriente, sugerindo, com isto, uma forte relação de pastagem do zooplâncton sobre o fitoplâncton (Bidigare, 1983). Segundo Esnal \& Daponte (1999), o comportamento de migração vertical de salpas pode estar relacionado também com a concentração destes organismos em superfície para o processo de liberação de gametas na fase sexuada.

Os experimentos das taxas de filtração mostram uma alta variação entre os frascos teste. Os valores das taxas variaram de 25,4 a 465,1 mL. dia ${ }^{-1}$ com uma média de 176,4 $\mathrm{mL}$.dia-1 (Tabela 1). Os valores obtidos estão dentro do intervalo observado por outros autores, como Parsons et al. (1984) de 192 $\mathrm{mL} \cdot \mathrm{dia}^{-1}$ e entre 82 a $444 \mathrm{~mL} \cdot \mathrm{dia}^{-1}$ segundo Vargas \& Madin (2004). Esta taxa individual de filtração de T. democratica equivale, no mínimo, a 20 copépodas, indicando um forte componente na ecologia pelágica do sistema (Parsons et al., 1984).

Para as taxas de crescimento, observou-se que os oozooides incubados não apresentaram crescimento durante o período de incubação, mas foi observado uma liberação média de 36 blastozooides por oozooides e com taxas de crescimento variando de 8 a 10,3\% em comprimento por hora o que equivale a mais de $30 \%$ de crescimento médio em peso (Tabela 1). Heron (1972a), em seu clássico trabaIho com $T$. democrática, ressalta taxas de crescimento ( $\mathrm{mm} /$ hora) em laboratório superiores a $10 \%$ sendo um dos maiores observados entre os invertebrados.

Apesar de não terem sido observados processos de ressurgência durante o fundeio, os resultados apresentados neste trabalho indicam o potencial de filtração e as altas taxas de crescimento de $T$. democratica na plataforma sul do Brasil. A ocorrência de manchas destes organismos, como ressaltado por Meneghetti (1973) e Pires-Vanin et al. (1993) apresentam uma explicação fisiológica de adaptação em crescimento individual e populacional, seja ela voltada como defesa a predação (Heron, 1972a), ou como um oportunismo frente a competidores herbívoros.

\section{AGRADECIMENTOS}

Os autores agradecem ao CEPSUL/ IBAMA pela oportunidade de uso do Navio de Pesquisa Soloncy Moura e a toda sua tripulação, assim como a Universidade do Vale do Itajaí por disponibilizar recursos para a saída da embarcação.

\section{REFERÊNCIAS}

Amaral, W.J.A. 1996. Composição, distribuição e abundância de salpidae (Thaliacea) na plataforma brasileira entre as latitudes 24( S e 29( 42' S - SUESTE I. Monografia. Curso de Especialização em Ecologia Aquática Costeira. Depto. Oceanografia. FURG. 16pp.

Amaral, W.J.A.; Montú, M.A. \& I.M. Gloeden. 1997. Salpidae (Thaliacea) da plataforma continental do extremo sul do Brasil: com- 
posição, distribuição e abundância (verão de 1990). Atlântica, 19:51-66.

Andersen, V. \& P. Nival. 1988. A pelagic ecosystem model simulation production and sedimentation of biogenic particles: role of salps and copepods. Mar. Ecol. Prog. Ser. 44:37-50.

Barlow, R.G., Mantoura, F.A., Gouch, M.A. \& T.W. Fileman. 1993. Pheopigment distribution during the 1990 spring bloom in the north-eastern Atlantic. Deep-Sea Res., 40: 2229-2242.

Beers, J.R. 1976. Determination of zooplankton biomass. In: H.F. Steedman (ed.), Zooplankton fixation and preservation. UNESCO, Paris. pp. 35-84.

Bidigare, R.R. 1983. Nitrogen excretion by marine zooplancton. In: Carpenter, E.J. \& D.G. Capone (eds.) Nitrogen in the marine environment. Academic Press, New York, 385-409.

Blackburn, M. 1979. Thaliacea of California Current region: realations to temperature, chlorophyll, currents and upwelling. CalCOFI Rep. 20,184-212.

Brandini, F.P.; Lopes, R.M.; Gutseit, K.S.; Spach, H.L. \& R. Sassi. 1997. Planctonologia na plataforma continental do Brasil. Diagnose e revisão bibliográfica. REVIZEE. 196pp.

Carvalho, J.L.B.; Schettini, C.A.F. \& T.M. Ribas. 1998. Estrutura termohalina do litoral centro-norte catarinense. Notas Téc. FACIMAR, 2:181-197.

Esnal, G.B. \& M.C. Daponte. 1999. Salpida. In: Boltovskoy, D. South Atlantic Zooplankton. Backhuys Publ. Leiden. 2:1423-1444 pp.

Esnal, G.B. 1981. Thaliacea: Sapidae. In: Boltovskoy, D. (ed.), Atlas del zooplancton del Atlantico Sudoccidental y métodos de trabajo con el zooplancton marino. INIDEP, Publ. Esp. Mar del Plata. Argentina. pp. 793-808.

Fernandes, L.M. 1998. Distribuição e abundância de Cladocera e Thaliacea na região e proximidades da Reserva Biológica Marinha do Arvoredo (Santa Catarina - Brasil). Monografia, Univ. Algarve, Faro. 46pp.

Gibbs, W.W. 1995. Some like it hot. Thriving tunicates may help clear the air of excess $\mathrm{CO}_{2}$. Scient. Am. December. 20-21.

Heron, A.C. 1972a. Population ecology of a colonizing species: the pelagic tunicate Thalia democratica. I individual growth rate and generation time. Oecologia, 10,269293.

Heron, A.C. 1972b. Population ecology of a colonizing species: the pelagic tunicate Thalia democratica. II population growth rate. Oecologia, 10,294-312.

Meneghetti, J.O. 1973. Zooplankton from southern Brazil - 1. Quantitative aspects. Iheringia. Zoo., 43,60-74.

Omori, M. \& T. Ikeda. 1984. Methods in marine zooplankton ecology. John Wiley \& Sons Publ., New York. 332p.

Parsons, T.R.; Takahashi, M. \& B. Hargrave. 1984. Biological Oceanographic Processes. 3rd Ed. Pergamon Press, Oxford. 330 pp.

Pires-Vanin, A.M.S., Rossi-Wongtschowski, C.L.D.B., Aidar, A., Mesquita, H.S.L., Soares, L.S.H., Katsuragawa, M. \& Y. Matsuura. 1993. Estrutura e função do ecossistema da plataforma continental da região de Ubatuba, São Paulo: síntese dos resultados. Bol. Inst. Oceanogr. Univ. São Paulo, 10,217-231.

Proença, L.A.O. 2002. Clorofila a do fitoplâncton em seis enseadas utilizadas para o cultivo de moluscos bivalves no litoral de Santa Catarina. Notas Téc. FACIMAR, 6:33-44.

Resgalla Jr., C. De La rocha, C. \& M. Montú. 2001. The influence of Ekman transport on zooplankton biomass variability off southern Brazil. J. Plank. Res. 23(6):641-650.

Strickland, J.D.H. \& T.R. Parsons. 1972. A practical handbook of seawater analysis, Bull. Fish. Res. Bd. Canada. 167pp.

Valentin, J.L.; Gaeta, S.A.; Sapch, H.L.; Montú, M.A. \& C. Odebrecht. 1994. Diagnóstico 
ambiental oceânico e costeiro das regiões sul e sudeste do Brasil. Volume 4. Oceanografia Biológica: Plâncton.

Vargas, C.A. \& L.P. Madin. 2004. Zooplankton feeding ecology: clearance and ingestion rates of the salps Thalia democratica, Cyclosalpa affinis and Salpa cylindrical on naturally occurring particles in the MidAtlantic Bight. J. Plank. Res. 26(7):827-833.
Wright, S. W., Jeffrey, S. W., Mantoura, F. A., Llewellyn, C. A., Bjornland, T., Repeta, D. \& N. Whelsmeyer. 1991. Improved HPLC method for the analysis of chlorophylls and carotenoids from marine samples. Mar. Ecol. Progr. Ser. 77:183-196. 